\title{
UV-Schutz: So geht's richtig!
}

Bei Kindern ist Hautkrebs zwar extrem selten, weil aber der Grundstein dafür im frühen Lebensalter gelegt wird, kommt dem Pädiater eine führende Rolle bei der Prävention zu. Richtiger Sonnenschutz ist das A und O - zum "Richtig“ gibt es allerdings eine Reihe von Irrtümern und Fehlschlüssen.

Die UV-induzierten Hauttumoren werden grob in zwei Kategorien eingeteilt. Am häufigsten sind die Basalzellkarzinome (Basaliome) und Plattenepithelkarzinome (Spinaliome), die unter dem Begriff „Non-Melanoma-Skin Cancer“ (NMSC) oder „weißer Hautkrebs“ zusammengefasst werden. Seltener, aber wesentlich gefährlicher, sind die Melanome, der sogenannte „schwarze Hautkrebs“.

\section{UV-Licht ist ein totales Karzinogen}

Der essenzielle Unterschied zwischen den beiden Tumortypen ist die Rolle der UV-Strahlung. Beim NMSC kommt es auf die kumulative Belastung im Laufe des Lebens an, egal ob mit oder ohne Sonnenbrände. Relevant sei allein die Gesamtdosis, präzisierte Prof. Dr. Thomas Schwarz von der Klinik für Dermatologie an der Universität Kiel. Daher könne es im Prinzip jeden treffen, wenn er nur lange genug lebe.

Beim Melanom sind die Zusammenhänge komplexer. Von Bedeutung ist weniger die kumulative als die episodische Belastung. Man weiß heute beispielsweise, dass mit der Zahl der Sonnenbrände in den ersten zwanzig Lebensjahren das Erkrankungsrisiko linear zunimmt und dass eine eindeutige Korrelation mit Solarienbesuchen besteht - je früher in der Jugend damit begonnen wird, desto höher das Risiko.

\section{Mythos "Sonnenschutzfaktor"}

Die beste Prävention ist ein ausreichender Sonnenschutz. Erste Säule ist für Schwarz vernünftiges Verhalten. So sollte man sich zwischen 10:30 und 14:30 Uhr im Schatten aufhalten, weil in dieser Zeit etwa zwei Drittel der gesamten Tagesstrahlung abgegeben wird. Schwarz erinnerte daran, dass die UVA-Einstrahlung auch bei bewölktem Wetter relativ hoch ist und Wasser - beispielsweise beim Schnorcheln - keineswegs wie häufig angenommen die UV-Strahlen völlig reflektiere. Die zweite Säule ist der textile Sonnenschutz inklusive Kopfbedeckung mit Krempe. Dabei spielt die Farbe keine Rolle, sondern relevant ist allein die Webdichte. Ob einwaschbare UV-Filter einen Nutzen haben, kann Schwarz nicht beurteilen. Für besonders starke und dauerhafte UV-Belastung wie beispielsweise beim Radfahren oder Surfen gibt es sogenannte „UV-Proof“-Kleidung. Sonnenschutzmittel kommen für Schwarz erst an dritter Stelle. Unterschieden wird zwischen physikalischen und chemischen Schutzfiltern, die die UV-Strahlung entweder reflektieren oder absorbieren. Wie stark eine Sonnencreme/-milch schützt, hängt vom Sonnenschutzfaktor (SPF) ab. Die Zahl errechnet sich aus dem Quotienten von minimaler Erythemdosis (MED) mit und ohne Filter. Ein SPF von 10 bedeutet beispielsweise, dass man ohne Hautrötung zehnmal länger in der Sonne bleiben kann als ohne Sonnencreme.
Welche Missverständnisse oder Wissensdefizite in punkto SPF bestehen verdeutlichte Schwarz sehr unterhaltsam mit einer Reihe von Rechenbeispielen. So filtert beispielsweise ein SPF 100 nicht wie häufig angenommen doppelt so viel UV-Strahlung wie ein SPF 50, sondern nur $1 \%$ mehr. Warum: SPF 10 lässt $10 \%$ der UV-Strahlung durch, SPF 50 nur $2 \%$ und SPF 100 nur noch $1 \%$. Einen SPF von 100 hält Schwarz auch aus anderen Gründen unsinnig. Wenn man 100-mal länger in der Sonne bleiben könne bis zum Auftreten eines Erythems (100-fache MED), dann seien das selbst bei sehr empfindlicher Haut, die schon nach zehn Minuten mit Rötung reagiere, schon etwa 16 Stunden (1.000 Minuten), bei einer MED von 20 sogar 33 Stunden!

\section{Schutzwirkung hängt von der aufgetragenen Menge ab}

Um den Stellenwert des SPF richtig einzuordnen, sollte man wissen, dass für den Test pro $\mathrm{cm}^{2}$ Haut $2 \mathrm{mg}$ Sonnencreme aufgetragen werden. Auch hier lohnt es sich nach Dafürhalten von Schwarz einmal nachzurechnen. Ausgehend von der Annahme, dass die Körperoberfläche eines Erwachsenen etwa $2 \mathrm{~m}^{2}$ $\left(20.000 \mathrm{~cm}^{2}\right)$ beträgt, werden für einmal „SPF-gerechtes“ Eincremen etwa $40.000 \mathrm{mg}$ (40 g) benötigt - das entspricht fast dem Viertel einer Flasche. Wer macht das schon? Trage man nur die halbe Menge ( $1 \mathrm{mg} / \mathrm{cm}^{2}$ ) einer Sonnencreme mit SPF 30 oder SPF 50 auf, dürfe man sich aber nicht darauf verlassen, dass damit immerhin noch der halbe Schutz erreicht werde, wies Schwarz auf einen weiteren immer noch nicht ausgerotteten Irrtum hin. SPF und Menge korrelierten nämlich nicht linear, sondern expotentiell. Man dürfe also nicht dividieren, sondern müsse die Wurzel ziehen - dann reduziere sich der SPF nicht auf 15 oder 25 , sondern auf 5 oder 7 .

\section{So viel auftragen, dass es fast unangenehm ist!}

Es werden keine besseren Sonnenschutzfaktoren gebraucht, sondern die verfügbaren Sonnenschutzmittel müssen nur dick genug aufgetragen werden. Dann reicht auch die einmal tägliche Anwendung aus, wie bei einer dänischen Untersuchung herauskam. Nach acht Stunden körperlicher Aktivität inklusive Baden war noch $43 \%$ des SPF vorhanden [1].

Schwarz rät seinen Patienten, soviel aufzutragen, dass es ihnen fast unangenehm ist. Wichtig war Schwarz auch der Hinweis, dass es einen „Sonnenschutz von Innen“ mit Vitaminen, Spurenelementen oder ähnlichem nicht gibt! (Gabriele Blaeser-Kiel)

[1] Bodekaer M et al. Photodermatol Photoimmunol Photomed 2008; 24: 296-300;

Hauttumore bei Kindern. Klinik und Prävention. 108. Jahrestagung der Deutschen Gesellschaft für Kinder- und Jugendmedizin (DGKJ), Hamburg 